\title{
A polymer-based surface grating coupler with an embedded $\mathrm{Si}_{3} \mathrm{~N}_{4}$ layer
}

\author{
Linghua Wang, ${ }^{1,2,3}$ Yanlu Li, ${ }^{1,3}$ Marco Garcia Porcel, ${ }^{1,3}$ Diedrik Vermeulen, ${ }^{1,3}$ Xiuyou Han, ${ }^{2}$ \\ Jinyan Wang, ${ }^{4}$ Xigao Jian, ${ }^{4}$ Roel Baets, ${ }^{1,3}$ Mingshan Zhao, ${ }^{2, a)}$ and Geert Morthier ${ }^{1,3}$ \\ 'Photonics Research Group, INTEC-Department, Ghent University-IMEC, Ghent B-9000, Belgium \\ ${ }^{2}$ School of Physics and Optoelectronic Technology, Dalian University of Technology, Dalian 116023, China \\ ${ }^{3}$ Center for Nano- and Biophotonics (NB-Photonics), Ghent University, Ghent B-9000, Belgium \\ ${ }^{4}$ Department of Polymer Science \& Materials, Dalian University of Technology, Dalian 116023, China
}

(Received 1 March 2012; accepted 30 April 2012; published online 5 June 2012)

\begin{abstract}
Polymer has been considered to be an ideal material option for integrated photonics devices. To measure these devices, normally the route of edge coupling is chosen to couple the light into or out of the polymer waveguide, which, however, demands a more difficult alignment procedure compared to the surface coupling. Due to the relatively low refractive indices of polymers, implementing the surface grating coupler for surface coupling in this material system remains a challenge. In this paper, we present a polymer-based surface grating coupler. Rather than through expensive complementary metal-oxide-semiconductor (CMOS) fabrication, the device is fabricated through a simple and fast UV-based soft imprint technique utilizing self-developed low-loss polymer material. The coupling efficiency is enhanced by embedding a thin $\mathrm{Si}_{3} \mathrm{~N}_{4}$ layer between the waveguide core and under cladding layer. Around $-19.8 \mathrm{~dB}$ insertion loss from singlemode fiber (SMF) to single-mode fiber is obtained for a straight waveguide with a grating coupler at each end. If the output light is collected with a multimode fiber, the insertion loss can be reduced to around $-17.3 \mathrm{~dB}$. Near $12 \%$ of the coupling efficiency is achieved between the polymer waveguide and SMF. The $3 \mathrm{~dB}$ bandwidth of the transmission is $32 \mathrm{~nm}$ and is centered at $1550 \mathrm{~nm}$. The proposed surface grating coupler and its easy fabrication method would be very attractive for practical applications. (C) 2012 American Institute of Physics. [http://dx.doi.org/10.1063/1.4724335]
\end{abstract}

\section{INTRODUCTION}

Recently, biological and chemical sensors have attracted lots of attention due to their vast applications in the fields of food safety and environmental monitoring, point-of-care diagnostics, drug discovery, and so on. Among these different types of sensors, planar integrated photonic biosensors have distinct advantages. Using photonic biosensors as transducer and detecting their output optical signal, enables labelfree and real-time monitoring of the dynamics of molecules' reactions. The sensor can be designed as a planar cavity structure, such as a ring or disk resonator, for example, to reduce the footprint while its sensitivity can be greatly enhanced. ${ }^{1,2}$ Fabricating photonic biosensors with mature techniques such as CMOS based processes or novel nanoimprint lithography further reduces the chip cost with high volume production. ${ }^{3,4}$

Besides the advantages mentioned above, compact photonic biosensors with different functionalized surfaces can be accommodated onto a single chip, which makes multiplexed sensing possible. This has been realized within the SOI (silicon-on-insulator) platform through the well known surface grating coupler. ${ }^{5,6}$ The output signals from different sensing channels are vertically coupled into free space and collected by an infrared camera. Besides the SOI platform, polymer has also been considered to be an ideal material platform for photonic biosensors, with the

"i)Email: mszhao@dlut.edu.cn. advantages such as extremely low cost, biocompatibility, etc., ${ }^{7.8}$ The polymer based photonic biosensors can perform as well as their counterparts in SOI with similar design structures. $^{9-12}$ However, a surface grating coupler is difficult to implement in this material platform because of the low refractive index contrast, ${ }^{13,14}$ which limits the application of photonic biosensors based on polymer for multiplexed sensing. In contrast, the route of edge coupling is usually chosen in order to couple the light into or out of the polymer waveguide, in which case the requirement for good waveguide facets is high. ${ }^{15}$ Another problem for edge coupling is the small tolerance on the alignment. For single-mode polymer waveguides, the measured power could fall dramatically with a few micrometers fiber drift. In order to circumvent these problems, we propose a grating coupler built on the polymer platform. Rather than through expensive CMOS fabrication, the device is fabricated through a simple UV based soft imprint technique utilizing self-developed low loss polymer material. The coupling efficiency is enhanced by embedding a thin $\mathrm{Si}_{3} \mathrm{~N}_{4}$ layer between the waveguide core and under cladding layer. Around $-19.8 \mathrm{~dB}$ insertion loss of single-mode fiber (SMF) to SMF is obtained for a straight waveguide with grating couplers at each end, which can be reduced to $-17.3 \mathrm{~dB}$ if the output light is collected by multimode fiber (MMF). The result indicates that the proposed grating coupler has a coupling efficiency of around $12 \%$ between SMF and polymer waveguide. The $3 \mathrm{~dB}$ bandwidth of the transmission spectrum is $32 \mathrm{~nm}$ centered at $1550 \mathrm{~nm}$. 


\section{PROPOSED STRUCTURE AND SIMULATION}

As mentioned before, the fundamental reason why the surface grating couplers cannot obtain a high coupling efficiency is the low refractive index of the polymer. The perturbation from the grating structures on the polymer waveguide surface has a limited influence on the waveguide mode, such that most of the light transmits directly through the waveguide instead of being diffracted out of the plane. The upward radiated power can only be increased on the condition that this perturbation is strengthened. At the same time, the field profile must also be optimized in order to increase the coupling to the fiber.

The proposed structure is depicted in Fig. 1. The grating pattern is fabricated on the under cladding layer, which sits on top of the Si substrate. A high refractive index layer, $\mathrm{Si}_{3} \mathrm{~N}_{4}$ in our case, is selectively deposited on the grating and then embedded between the under cladding and waveguide core layer. The excess part of the $\mathrm{Si}_{3} \mathrm{~N}_{4}$ layer is removed from the waveguide to prevent additional loss.

The simulation is carried out with the combination of CAvity Modelling FRamework (CAMFR) (Ref. 16) and FDTD (finite-difference-time-domain). ${ }^{17}$ Parameters such as thickness of waveguide core, thickness of $\mathrm{Si}_{3} \mathrm{~N}_{4}$ layer, grating height, grating period, and so on are optimized to obtain suitable coupling angle, higher upward power, ideal diffracted field profile and thus higher coupling efficiency with the fiber. The electric field plots with and without the embedded high index $\mathrm{Si}_{3} \mathrm{~N}_{4}$ layer are shown in Fig. 2. The used parameters are as follows: thickness of waveguide core $\mathrm{d}=1.7 \mu \mathrm{m}$, grating height $\mathrm{e}=0.7 \mu \mathrm{m}$, thickness of $\mathrm{Si}_{3} \mathrm{~N}_{4}$ layer $\mathrm{t}=0.2 \mu \mathrm{m}$, grating period $\Lambda=1.7$ $\mu \mathrm{m}$. It can be clearly seen that after adding the $\mathrm{Si}_{3} \mathrm{~N}_{4}$ layer, the perturbation of the surface grating on the waveguide mode is strongly enhanced, resulting in more upward power with diffraction angle around $43^{\circ}$. Besides that, the near-field electric field starts exhibiting a quasi-Gaussian profile, which is more easily fitted to the standard Gaussian field profile of the optical fiber to have higher coupling efficiency. This is shown in Fig. 3. The spectral response of the grating with the optimized structure is shown in Fig. 4. It shows a maximum coupling efficiency with the single-mode fiber of around 19\%. Another benefit brought by this structure is polarization selectivity. The TM mode light cannot be coupled into or out of the polymer waveguide as effectively as the TE mode light, and only has an efficiency of less than $6 \%$.

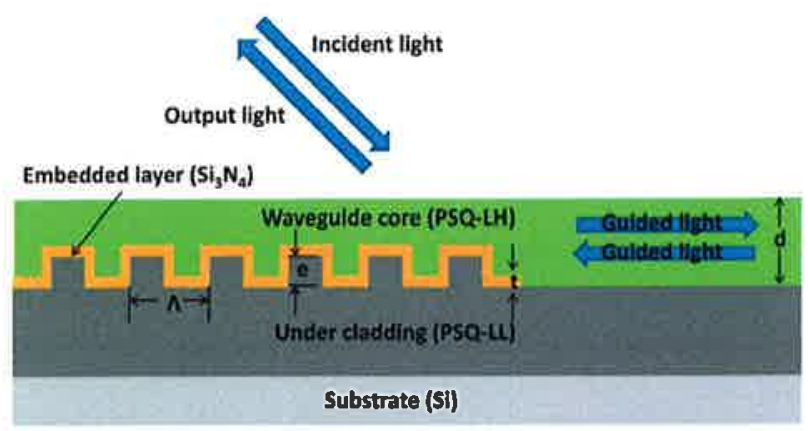

FIG. 1. The proposed polymer based surface grating coupler.
Diffracted fleid

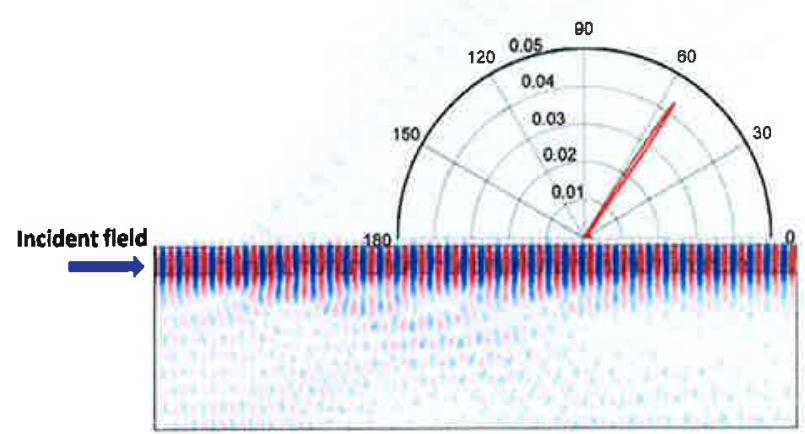

(a)

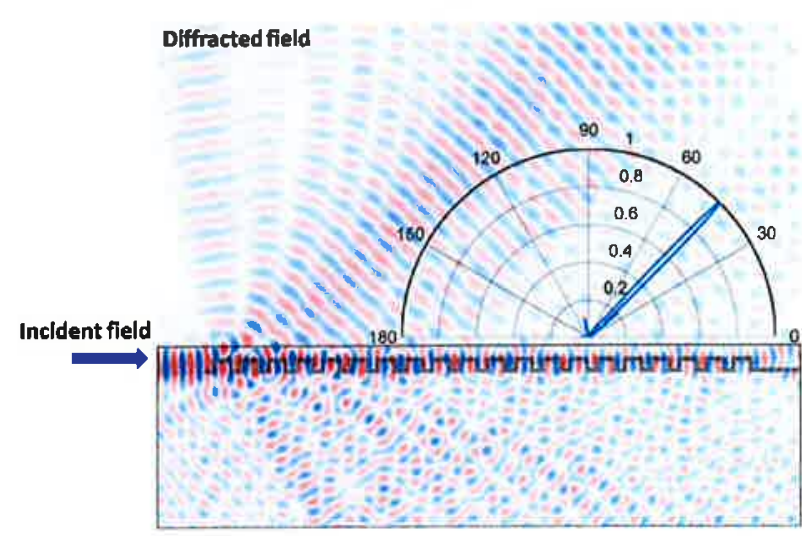

(b)

FIG. 2. The electric field plot without (a) and with (b) the embedded high index $\mathrm{Si}_{3} \mathrm{~N}_{4}$ layer.

\section{MATERIALS AND FABRICATION PROCESSES}

We developed a simple UV-based soft imprint technique to realize the structure proposed above. Compared with lithography and dry etching, this technique has been proved to be an efficient way to directly pattern the polymer waveguide and devices. However, this process has certain requirements

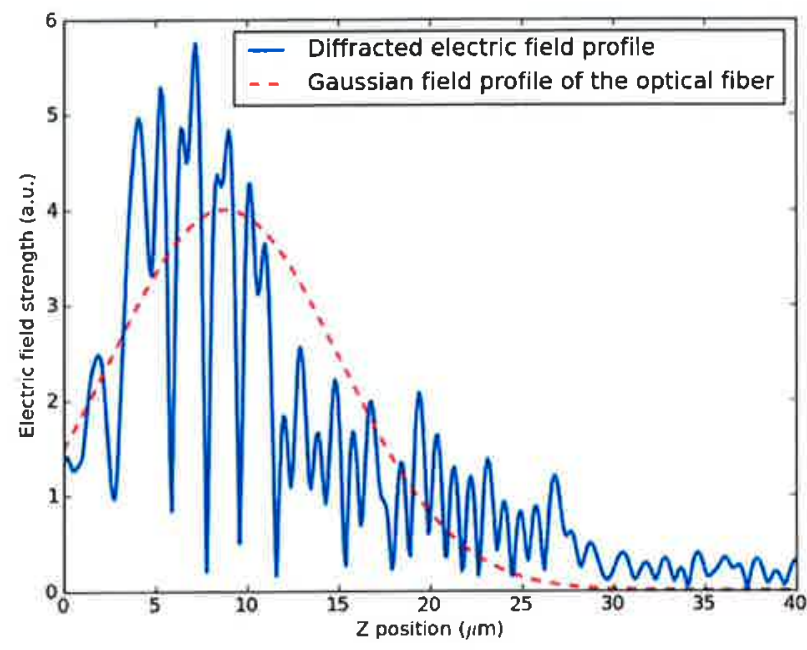

FIG. 3. The near field electric field profile of the proposed grating structure simulated by CAMFR. 


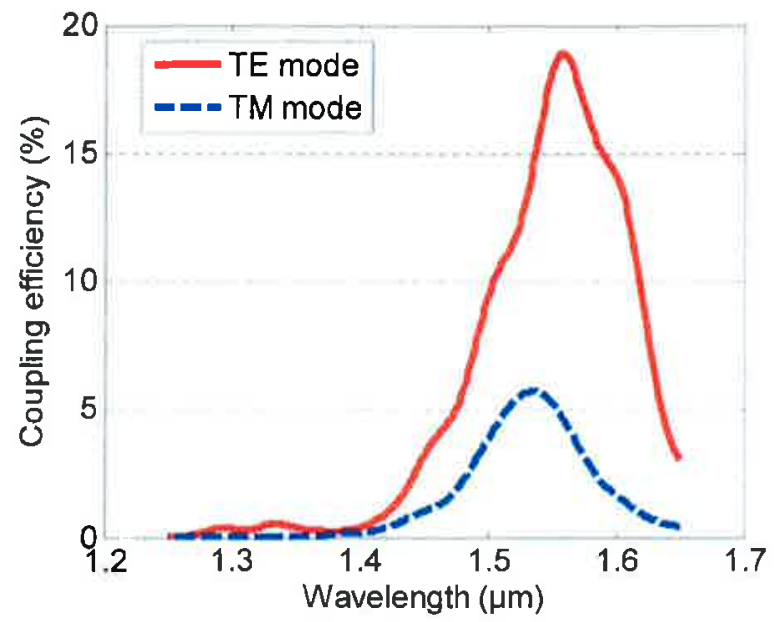

FIG. 4. The spectrum response of the proposed grating structure simulated by FDTD.

on the imprinted material. Besides good optical properties such as low optical loss, low birefringence and high thermal stability, this process also requires good UV curing and low viscosity. The material we used for this work, which is a kind of liquid polysilisiquioxane (PSQ) resin, meets the requirements above. Its two components, PSQ-LH with high refractive index $(\mathrm{n}=1.52)$ and PSQ-LL with low refractive index $(n=1.45)$, are used as the waveguide core and cladding, respectively. ${ }^{18}$ The fabrication process is shown in Fig. 5. This process starts with the fabrication of the pattemed mold. The polydimethylsiloxane (PDMS) soft mold is replicated from a master mold on which the grating and waveguide patterns are defined by the contact lithography. The obtained soft mold is then pressed against the under cladding layer, which is spin-coated on the silicon wafer. After 3 min UV light curing with an intensity of around $2000 \mathrm{~mJ} / \mathrm{cm}^{2}$, the soft mold is peeled from the substrate, leaving the grating and waveguide patterns on the under cladding layer. This layer is fully cured by $2 \mathrm{~h}$ of $180^{\circ} \mathrm{C}$ thermal curing. In order to realize the selective $\mathrm{Si}_{3} \mathrm{~N}_{4}$ embedded structure, first the previously obtained sample is deposited with $\mathrm{Si}_{3} \mathrm{~N}_{4}$ through PECVD (plasma-enhanced chemical vapor deposition). This step needs to be carefully optimized because our polymer cannot resist too high temperature (above $200{ }^{\circ} \mathrm{C}$ ) during deposition. However, using too low temperature is detrimental to this dielectric layer, especially since its refractive index drops dramatically, which would degrade the performance of the grating. The detailed characterization process for $\mathrm{Si}_{3} \mathrm{~N}_{4}$ layer deposition is shown in Fig. 6, where the film thickness and refractive index value are measured by ellipsometer. By experimenting, the deposition temperature was chosen to be $150{ }^{\circ} \mathrm{C}$, at which the deposition rate and refractive index are found to be $12.72 \mathrm{~nm} / \mathrm{min}$ and 1.84 , respectively. Targeted $200 \mathrm{~nm}$ layer thickness was achieved with a deposition time of around $17 \mathrm{~min}$. Rather than dry etching, a simple wet etching method with a Hydrofluoric acid $(\mathrm{HF})$ solution was used to completely remove the $\mathrm{Si}_{3} \mathrm{~N}_{4}$ layer from the waveguide region, leaving only the grating area covered by the $\mathrm{Si}_{3} \mathrm{~N}_{4}$. After the spin coating and curing of the core layer PSQ-LH, the proposed surface grating coupler structure was realized. The microscope and SEM images of the fabricated device are shown in Figs. 7(a) and 7(b).

\section{MEASUREMENT RESULTS}

The straight waveguide with a surface grating coupler at each end is measured with a vertical setup. On this setup, a cleaved single-mode fiber connected to a tunable laser through the polarization controller was used to launch the light. The light diffracted by the surface grating coupler at the output was collected by either a SMF or an MMF, which was connected to an optical power meter. The input and output fibers are mounted on high-precision 3-axis translation stages, on which they can be tuned between $36^{\circ}$ and $45^{\circ}$ with respect to the vertical direction using a rotation arm. The sample is mounted on a vacuum chuck on a two-axis stage.

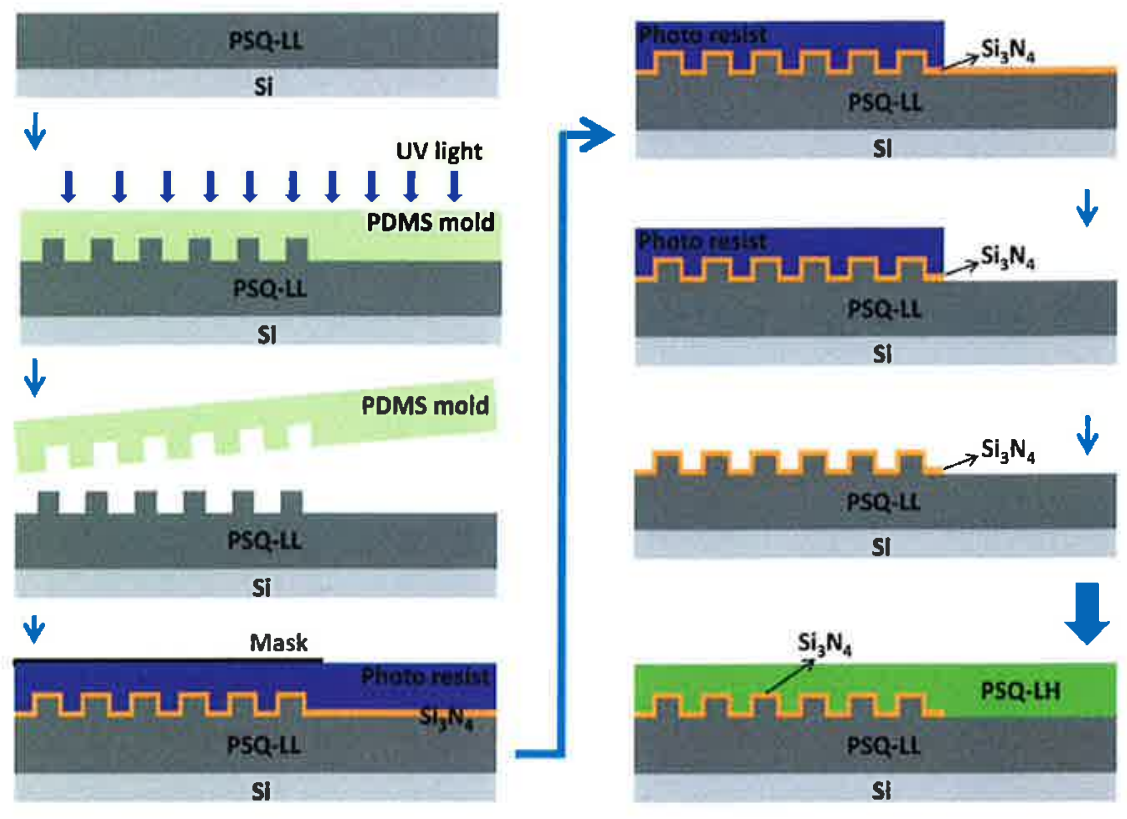

FIG. 5. The fabricating process of UV soft imprint lithography combined with wet etching to realize the proposed structure. 


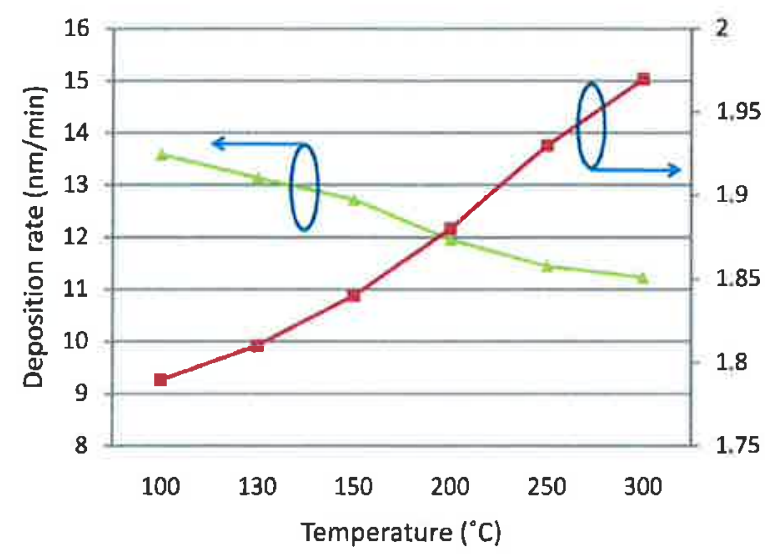

FIG. 6. The characterization process for the $\mathrm{Si}_{3} \mathrm{~N}_{4}$ layer deposition.

Both the tunable laser and power meter are controlled by a computer. The measurements result is shown in Fig. 8. The optimal coupling angle was found to be $40^{\circ}$. At this angle, around $-19.8 \mathrm{~dB}$ SMF to SMF insertion loss is obtained. We measured the straight waveguide loss with a similar inverted-rib structure through Fabry-Perot resonance method before and it was found to be $1.7 \mathrm{~dB} / \mathrm{cm} .{ }^{19}$ In this case, the length of the straight and tapered waveguides connecting the two surface grating couplers is $5 \mathrm{~mm}$, resulting in an estimated transmission loss of $0.85 \mathrm{~dB}$. The pure link loss is measured to be $0.42 \mathrm{~dB}$ at $1550 \mathrm{~nm}$ wavelength. Thus, the coupling efficiency for each surface grating coupler can be

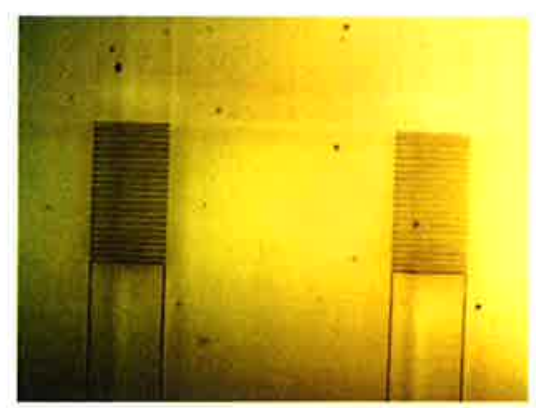

(a)

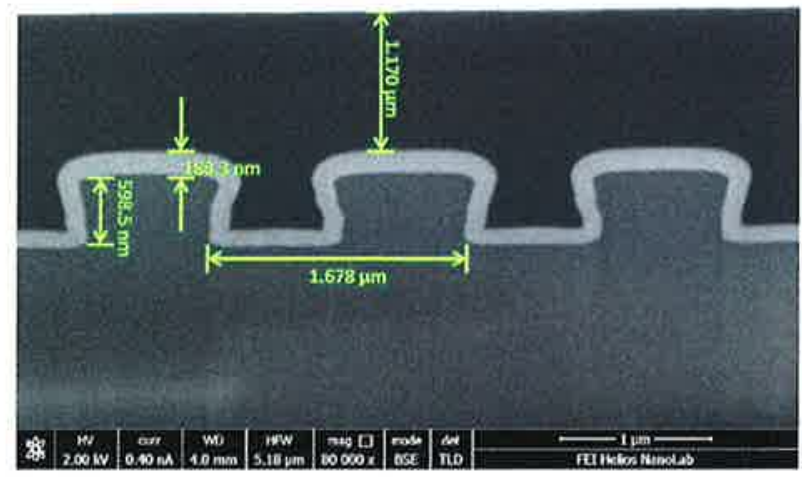

(b)

FIG. 7. The microscope (a) and SEM (b) picture of the fabricated surface grating coupler with $\mathrm{Si}_{3} \mathrm{~N}_{4}$ layer embedded between the core and under cladding layer.

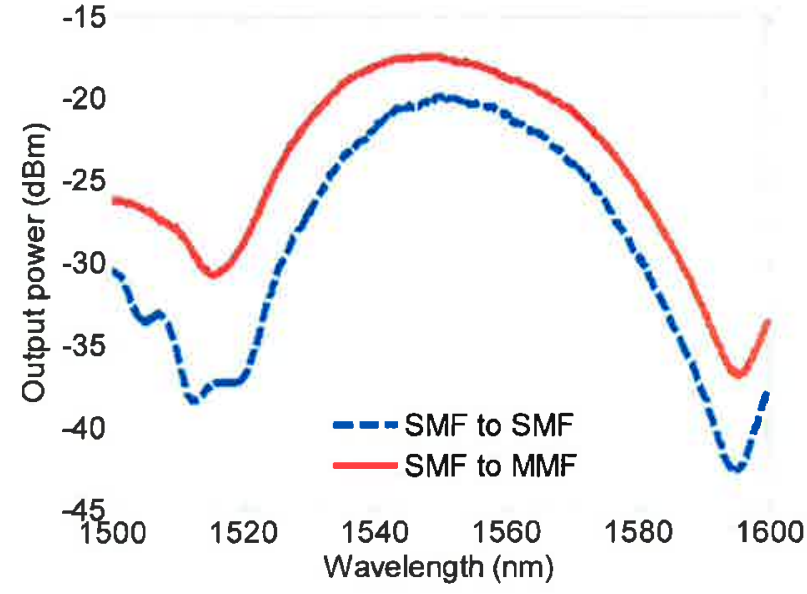

FIG. 8. The measused fiber to fiber transmission spectrum of the straight waveguide with a grating coupler at each end.

determined to be $-9.27 \mathrm{~dB}$ (nearly $12 \%$ ) conservatively. There are another two factors we still have not taken into account because they are currently difficult to predict. One is the junction loss from the grating coupler to the waveguide and the other is the surface reflection loss. Considering this, the actual coupling efficiency can be even higher. The $3 \mathrm{~dB}$ bandwidth is $32 \mathrm{~nm}$ centered at $1550 \mathrm{~nm}$. MMF was also used to receive the output power. Because of the large aperture of the MMF, the total SMF to MMF insertion loss was reduced by $2.5 \mathrm{~dB}$, which is $-17.3 \mathrm{~dB}$.

In order to find out how the energy is distributed within the first order of the proposed surface grating coupler, we carefully tune the angle of the output SMF while keeping that of the input SMF at $40^{\circ}$. The measured result is shown in Fig. 9. The $3 \mathrm{~dB}$ angle bandwidth is around $7^{\circ}$. This result confirms that the first order is the main order of such grating coupler. The small energy distributed angle also shows the possibility of imaging the output light onto an infrared camera in the far field.

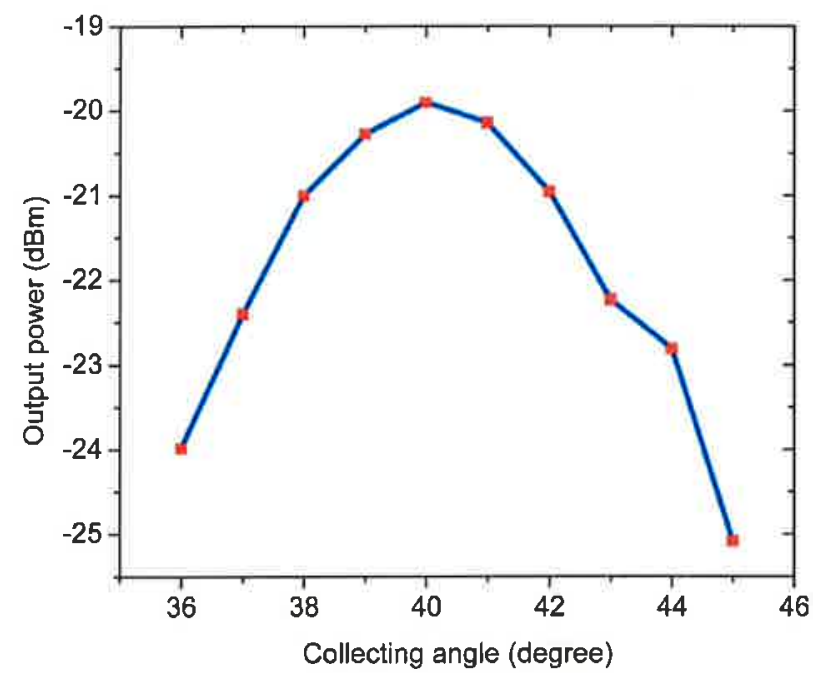

FIG. 9. The changing of the maximum output power with the angle of the SMF at the output end. 


\section{v. CONCLUSION}

In this paper, a surface grating coupler based on a polymer platform is proposed. A high refractive index layer of $\mathrm{Si}_{3} \mathrm{~N}_{4}$ is embedded between the under cladding and waveguide layer to obtain good directionality and higher coupling efficiency with the fiber. Rather than expensive CMOS fabrication, the device is fabricated through a simple UV-based soft imprint technique utilizing self-developed low loss polymer material. With a single-mode fiber, the coupling efficiency is around $12 \%$. The $3 \mathrm{~dB}$ bandwidth centered at 1550 $\mathrm{nm}$ is $32 \mathrm{~nm}$. The proposed structure would be very attractive in applications that require out-of-plane coupling or multiple routes signal processing.

\section{ACKNOWLEDGMENTS}

The authors would like to thank Steven Verstuyft and Liesbet Van Landschoot's help on the fabrication and characterization. Linghua Wang acknowledges the support by the Chinese Scholarship Council (CSC) and by BOF co-funding from Ghent University. He also appreciates the helpful discussions with Dr. Zhechao Wang and Dr. Ananth Subramanian. This work is also supported in part by National High-tech R\&D Program (Grant No. 2012AA040406) and National Natural Science Foundation of China under Grant Nos. 61077015, 60807015, and 60577014.
${ }^{1}$ K. De Vos, J. Girones, S. Popelka, E. Schacht, R. Baets, and P. Bienstman, J. Biosens. Bioelectron. 24, 2528 (2009).

${ }^{2}$ D.-X. Xu, M. Vachon, A. Densmore, R. Ma, S. Janz, A. Delâge, J. Lapointe, P. Cheben, J. H. Schmid, E. Post, S. Messaoudène, and J.-M. Fédéli, Opt. Express 18, 22867 (2010).

${ }^{3}$ L. Jay Guo, Adv. Mater 19, 495 (2007)

${ }^{4}$ J. K. S. Poon, Y. Y. Huang, G. T. Paloczi, and A. Yariv, IEEE Photon, Technol. Lett 16, 2496 (2004)

${ }^{5}$ K. De Vos, J. G. Molera, T. Claes, Y. De Koninck, S. Popelka, E. Schacht, R. Baets, and P. Bienstman, IEEE Photon. J, 1, 225 (2009).

${ }^{6}$ G. Roelkens, D. Vermeulen, D. Van Thourhout, R. Baets, S. Brision, P. Lyan, P. Gautier, and J.-M. Fedeli, Appl. Phys. Lett. 92, 131101 (2008).

${ }^{7}$ H. Ma, A. K.-Y. Jen, and L. R. Dalton, Adv. Mater 14, 1339 (2002)

${ }^{8}$ L. Eldada, Proc. SPIE 6351, 63510Y (2006)

${ }^{9}$ C. Y. Chao and L. J. Guo, IEEE J. Sel. Topics Quantum Electron. 12, 134 (2006).

${ }^{10} \mathrm{~J}$. B. Wright, I. Brener, K. R. Westlake, D. W. Branch, M. J. Shaw, and G. A. Vawter, Proc, SPIE 7605, 76050C (2010),

${ }^{11}$ R. Bruck, E. Melnik, P. Muellner, R. Hainberger, and M. Lämmerhofer, J. Biosens. Bioelection. 26, 3832 (2011).

${ }^{12}$ J.-W. Kim, K.-J. Kim, J.-A. Yi, and M.-C. Oh, IEEE J. Sel, Top. Quantum Electron. 16, 973 (2010).

${ }^{13}$ R. Waldhäusl, B. Schnabel, P. Dannberg, E.-B. Kley, A. Bräuer, and W. Karthe, Appl. Opt. 36, 9383 (1997).

${ }^{14} \mathrm{R}$. Bruck and R. Hainberger, Appl. Opt. 49, 1972 (2010)

${ }^{15}$ T. Han, S. Madden, D. Bulla, and B. L.-Davies, Opt. Express 18, 19286 (2010).

${ }^{16}$ See http://camfr.sourceforge.net for CAMFR.

${ }^{17}$ K. S. Yee, IEEE Trans. Antennas Propag. 14, 302 (1966)

${ }^{18}$ H. Zhang, J. Wang, L. Li, Y. Song, M. Zhao, and X. Jian, Thin Solid Films 517, 857 (2008).

${ }^{19}$ J. Teng, H. Yan, L. Li, M. Zhao, H. Zhang, and G. Morthier, IET Optoelectron. 5, 265 (2011). 